\title{
El aula como sistema complejo: hacia una formalización de la organización de la vida en el aula
}

\section{The classroom as a complex system: towards a formalization of the organization life in the classroom}

\author{
Ronnie Alejandro Videla Reyes ${ }^{1}$, Guillermo Leyton García ${ }^{2}$, Sebastián Rossel Salas $^{3}$ \\ ${ }^{1}$ Departamento de Educación, Universidad de La Serena, Chile (ronnie.videla@gmail.com) \\ ${ }^{2}$ Departamento de Ingeniería en Computación, Universidad de La Serena, Chile (gleyton@ userena.cl) \\ ${ }^{3}$ Centro de Lenguaje, Universidad de La Serena, Chile (napellus@gmail.com)
}

Recibido el 9 de septiembre de 2017; revisado el 2 de noviembre de 2017; aceptado el 2 de noviembre de 2017; publicado el 1 de diciembre de 2017

\begin{abstract}
RESUMEN:
Avanzar hacia un concepto de educación que connote y defina lo educativo a partir de la dinámica que exhibe la organización del aprendizaje en el aula requiere del uso las ciencias de la complejidad. Asumir la teoría de sistemas dinámicos no-lineales para comprender los fenómenos de interacciones de aprendizaje como una historia de coherencias moleculares, sensoriales y relacionales es el gran desafío de las ciencias cognitivas. El presente estudio realiza una revisión teórica-empírica respecto a estudios en sistemas dinámicos basados en describir la organización de los seres vivos, la vida humana y la vida en el aula. Se propone que el aula es un sistema complejo de interacciones de aprendizaje donde la mediación semiótica actúa como homeostasis lingüística determinando el devenir del sistema. Particularmente se propone a la teoría fuzzy set como herramienta matemática de dinámica no-lineal para formalizar la organización del aprendizaje en el aula.
\end{abstract}

PALABRAS CLAVE: SISTEMAS COMPLEJOS, AUTOPOIESIS, EDUCACIÓN, INTERACCIONES DE CLASES, FUZZY SET

\begin{abstract}
:
Moving towards a concept of education that connotes and defines the educational from the dynamics exhibited by the organization of learning in the classroom requires the use of the sciences of complexity. Assuming the theory of non-linear dynamical systems to understand the phenomena of learning interactions as a history of molecular, sensory and relational coherence is the great challenge of the cognitive sciences. The present study makes a theoretical-empirical review of studies in dynamic systems based on describing the organization of living beings, human life and classroom life. It is proposed that the classroom is a complex system of learning interactions where the semiotic mediation acts as linguistic homeostasis determining the future of the system. In particular, fuzzy set theory is proposed as a mathematical tool of non-linear dynamics to formalize the organization of learning in the classroom.
\end{abstract}

$\begin{array}{lcr}\text { KEYWORDS: } \quad \text { COMPLEX } & \text { SYSTEMS, } \\ \text { AUTOPOIESIS, } & \text { EDUCATION, } & \text { CLASS } \\ \text { INTERACTIONS, FUZZY SET } & \end{array}$




\section{INTRODUCCIÓN}

\subsection{Hacia una organización de los seres vivos}

A mitad del Siglo XX la ciencia se preguntaba sobre qué se connota y describe de los procesos moleculares que los seres vivos tienen, de modo que la realización de su vivir puede ser abstraída a partir de la relación interna de sus componentes como unidad autónoma. En esta búsqueda incansable sobre la caracterización de lo vivo, científicos en todo el mundo buscaban qué es lo que cambiaba en la célula, mientras que los biólogos chilenos Maturana y Varela (1973) se preocuparon de qué es lo que se conservaba. Después de varios experimentos y simulaciones evidenciaron que lo que caracteriza y define el vivir del ser vivo es su condición de sistema autopoiético. La autopoiesis molecular corresponde a la unidad funcional de los seres vivos, la cual es considerada un sistema autónomo y cerrado, que puede producirse a sí misma a través de la red molecular que la define, conservando su identidad y estructura durante las diferentes perturbaciones recibidas a lo largo de su ontogenia. Esta propuesta revolucionó la ciencia en el mundo, pues a partir de un modelo matemático computacional inspirado en los trabajos de cibernética de Von Forester se pudo formalizar la dinámica que caracteriza lo vivo desde las teorías de sistemas dinámicos no-lineales, especificando cómo los seres vivos pueden conservar sus relaciones de existencia y por ende posibilitar el devenir estructural y existencial del organismo (Varela, 1980).

La característica de los sistemas autopoiéticos es que son sistemas cerrados en sí mismos, ya que están determinados en su estructura y, que aun cuando reciban perturbaciones del medio externo, estas no especifican al sistema, solo dinamizan y cambian su conectividad estructural (Maturana, 2011). Estos cambios en el comportamiento son dinámicos, producto de la historia de acoplamientos estructurales que los seres vivos despliegan en su ontogenia (Maturana, Mpodozis y Letelier, 1995). El aprendizaje se concibe desde esta teoría como el devenir de cambios estructurales acoplados al flujo del vivir, donde el sistema nervioso es una red de neuronas interactuantes que involucran el encéfalo y la médula espinal. Al respecto, Maturana y Varela (1973) sostienen que el aprendizaje solo ocurre a partir de la autoproducción de sus componentes, y la conservación de dichas relaciones determinadas en la ontogenia, se pueden describir en tres estados del sistema nervioso:

i. El organismo, incluyendo el sistema nervioso provee el espacio físico y químico para la autopoiesis de las neuronas, siendo, además, una fuente de perturbaciones físicas y químicas que pueden alterar estas y conducir al organismo a los estados i y ii.

ii. ii: Hay estados del organismo físicos y químicos que modulan la actividad del sistema nervioso completo, a partir de la acción de neuronas pre y post-sinápticas llevando al organismo al estado iii.

iii. Hay estados del sistema nervioso que cambian el estado del organismo físicos y químicos conduciéndolos a los estados i y ii. (Maturana y Varela, 1973, p. 122)

Esta transición de los tres estados del sistema nervioso que dan cuenta de la circularidad de transformaciones del organismo, establece que los seres vivos ocurren como entidades autopoiéticas moleculares dinámicas, autónomas y discretas, cuya realización continua es dependiente de su autoproducción molecular y que dicha conservación de estados no requiere de ningún principio organizador (Maturana y Dávila, 2015). El sistema nervioso carece de una teleología intrínseca y por ende persiste en ausencia de finalidad, quedando sujeto a las variaciones estructurales experimentados en el devenir de cada ser vivo (Froese y Fuchs, 2012).

Con el objetivo de formalizar la autopoiesis molecular y poder realizar una abstracción de las relaciones de autoorganizativas del sistema viviente, Varela, Maturana y Uribe (1974) propusieron, mediante un programa computacional, un modelo de reacciones químicas de síntesis y lisis, el cual daba cuenta de la dinámica de interacciones moleculares, cuya caracterización estaba regida por condiciones específicas de temperatura, originando espontáneamente la emergencia de unidades autopieticas como resultado del operar de sus componentes. El modelo obtenido estuvo definido a partir de las siguientes condiciones: sean A, M y B partículas y su operar, donde la cadena de $\mathrm{M}$, es flexible y movible y puede cerrarse sobre sí misma bajo las relaciones de catálisis, substrato y enlaces: 
1. $2 \mathrm{~B}+\mathrm{A} \rightarrow \mathrm{M}+\mathrm{A}$

2. $\mathrm{M}+\mathrm{M} \rightarrow \mathrm{MM}$

$\mathrm{MM}+\mathrm{M} \rightarrow \mathrm{MMM}$

3. $\mathrm{M} \rightarrow 2 \mathrm{~B}$

4. MMMMM es permeable al paso de B.

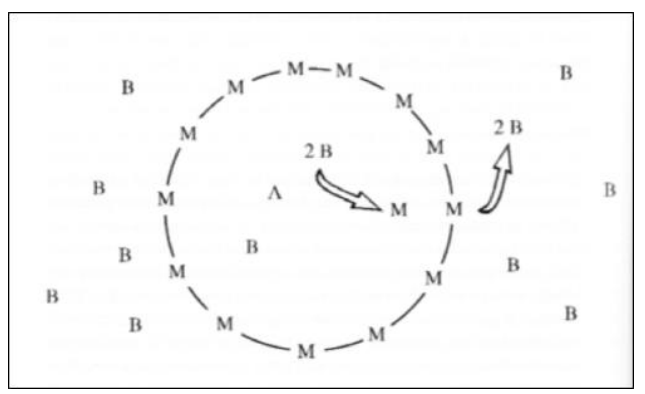

Figura 1: Formalización de la autopoiesis molecular (imagen extraída, Maturana y Varela, 1973).

A partir de este modelo se pudo evidenciar la relación generativa de la unidad autopoiética desde un dominio formal y abstracto no realizado antes. Este complemento a la teoría, que da cuenta de la dinámica interna que caracteriza los seres vivos, de modo que estos conservan su organización y por ende su existencia durante la ontogenia, está restringida solo a un nivel molecular de coherencias operacionales. Esta formalización permite connotar y describir lo vivo en tanto su dinámica es auto organizada. Sin embargo, cuando propone explicar y connotar la vida humana en distinción a otros seres vivos, es menester avanzar hacia una comprensión del fenómeno humano que integre la complejidad de las relaciones sociales entre sistemas autopoiéticos. En este sentido, se debe estudiar el flujo de descripciones de coherencias operacionales a nivel molecular, sensorial y relacional, las cuales se codeterminan como unidades ecológicas mediante lenguaje verbal y gestual (Maturana y Dávila, 2015).

\subsection{Hacia la organización de la vida humana}

La vida, en especial la vida humana, denota una complejidad mayor al momento en que se pretende dar cuenta de ella. Cada ser humano es una unidad ecológica que es catalizada por la relación indisociable entre organismo y nicho como expansión de la vida psicológica generativa del espacio cultural en donde se despliega (Maturana y Dávila, 2015). Cuando se establece una relación con un otro, por ejemplo, en un juego compartido como lanzarse una pelota, toda esta coordinación surge a partir de una congruencia relacional, la cual posibilita el decurso del juego donde estos participan, emergiendo espontáneamente una dinámica de interacción básica que está determinada por el devenir de intenciones mutuas en ausencia de finalidad (Maturana y Mpodozis, 2000). Al establecer un comportamiento social es menester el surgimiento de una respectividad o congruencia relacional entre los seres humanos. Es más, si queremos observar cuando dos seres humanos interactúan comunicativamente, el producto de esta interacción está urdido por información verbal y gestual. El devenir de esta dinámica comunicacional entre los seres humanos obedece a un nicho de respectividades mutuamente selectivas, cuya organización intersubjetiva permite la adaptación en y con el entorno (Froese, 2010). A este nicho de respectividad o congruencia relacional desde la teoría de los sistemas autopoiéticos se le denomina coderiva ontogénica:

Las interacciones recurrentes entre dos o más organismos dan origen a una coderiva estructural ontogénica. En un coderiva, los organismos interactuantes siguen derivas estructurales recíprocamente congruentes, y cada uno sigue el curso de cambios estructurales en que se conserva su organización y su adaptación en relación con otros, en un proceso que cursa hasta que alguno se separa o desintegra (Maturana y Mpodozis, 2000, p. 9).

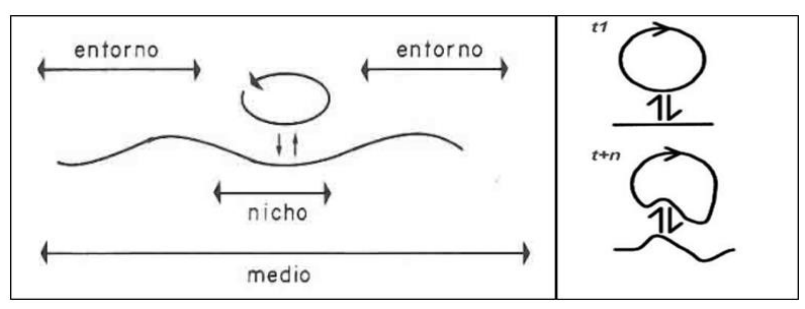

Figura 2: Coderiva ontogénica natural (imagen extraída de Maturana y Mpodozis, 2000)

A partir de la coderiva ontogénica se ha podido visualizar un campo fecundo para la comprensión de cómo las interacciones entre seres humanos permite establecer un nicho dinámico de entrelazamiento verbal y gestual. Por lo demás, se asume en la coderiva que la evolución de las relaciones está subordinada a la emergencia de ciertos dominios consensuales de respectividad, los cuales dependen primero de su condición como sistemas autopoiéticos y en segundo por el nicho de congruencia en la cual los comportamientos locales suscitan comportamientos globales del sistema (Thompson y Cosmelli, 2011). Cada ser humano, en su afán comunicacional con los otros y el mundo natural, establece una dinámica de interacciones, 
reguladas por un conjunto de aspectos contextuales, emocionales y cognitivos. En este ámbito sistémico de la interacción, las relaciones urdidas comprenden sistemas dinámicos, cuyo devenir transformacional del sistema está restringido a la evolución de los aspectos comunicativos, tanto en su despliegue temporal-espacial como linguístico (Fosa, Cornejo y Carré, 2016).

Uno de los escenarios interesantes para observar y analizar con profundidad la dinámica de interacción que rige la organización del sistema comunicacional a partir de múltiples niveles jerárquicos de despliegue y cambio de las relaciones entre seres humanos es el aula de clases (Mercer y Howe, 2012). Las interacciones que se tejen en un ámbito de educación formal están reguladas semióticamente por la conjugación de mecanismos de actividad intramental e intermental, en donde la dimensión meso personal y la dimensión macro cultural se sincronizan y des-sincronizan como resultado de una configuración histórica de encuentros y experiencias tamizadas por la génesis continua y cambiante de significados. La vida humana corresponde a la matriz de coherencias operacionales a nivel molecular, sensorial y relacional, la cual permite connotar a los seres humanos como seres biológico-culturales (Maturana y Dávila, 2015)

\subsection{Hacia la organización de la vida en el aula}

Es en el aula de clases donde el lenguaje como herramienta cultural permite establecer puentes de comunicación entre diferentes seres humanos en el tránsito de diversos espacios de interacciones, cuya dinámica es modulada por la intencionalidad comunicativa entre profesores-escolares y escolaresescolares (Mercer, 2010). La evidencia en el ámbito de interacción de clases ha reportado que las herramientas de andamiaje lingüístico tales como interrogación, feedback y explicaciones en torno al desarrollo de la tarea actúan como moduladores de las dinámicas de aprendizaje $\mathrm{y}$, por ende, exhiben patrones dinámicos específicos de transformaciones de la interacción comunicativa (Sánchez y Rosales, 2005). A partir de estas transformaciones de las interacciones comunicativas que se generan en la relación de sus componentes, se puede establecer que la organización de la vida en el aula está regulada por la mediación semiótica del aprendizaje, la cual corresponde a la identificación de un complejo de significados regulados por la homeostasis lingüística del turno comunicacional (Fossa, Cornejo y Carré, 2016). Este proceso de mediación semiótica se dinamiza en el espacio dialógico del aula, donde la varianza e invariancia de los significados es regulada por mecanismos específicos de interacción comunicativa entre profesores y alumnos y escolares con escolares. Este proceso sociogenético, en el cual se tejen las relaciones de cambio y transformación de los significados de aprendizajes, es producto de una realización histórica que no está determinada a priori, sino más bien, emerge.

$\mathrm{Si}$ el profesor, durante el decurso de la clase, interroga a los escolares del por qué han realizado una actividad de una manera particular y qué tipo de significados le asocian al contenido, es posible evidenciar una interacción comunicativa que fluirá en el tiempo exhibiendo una serie de transformaciones que dependerán exclusivamente del nivel de intersincronización de los componentes de la interacción, cuya co-construcción del significado no obedece a un tiempo específico asociado a una situación particular como un objetivo de aprendizaje, sino más bien emana permanentemente en la experiencia y connota un aprendizaje como síntesis de un espacio dialógico intersubjetivo. Este espacio dialógico intersubjetivo, como tejido comunicacional dentro del aula de clases, está mediatizado por la manera en que los profesores y escolares negocian y renegocian sus ideas.

La negociación y renegociación de ideas en las salas de clase varían según los actores del sistema y, por ende, el contexto temático y cultural en el cual se urden las relaciones. La co-construcción del significado en interacciones de clases, ha sido estudiada mayoritariamente desde el enfoque cognitivo sociocultural, imponiéndose como uno de los paradigmas contemporáneos más robustos a la hora de caracterizar la dinámica de interacción de aprendizaje entre profesores-escolares y escolaresescolares (Mercer y Howe, 2012). Mercer (1997; 2001; 2007) ha sido uno de los autores que más han contribuido a entender las interacciones comunicativas en el aula a partir de la investigación dialógica de situaciones diádicas, concibiendo el aprendizaje como un proceso de construcción comprehensiva, dejando atrás el sesgo individualista y promoviendo el estudio de reglas educativas básicas, generalmente implícitas, que permiten a los interlocutores alcanzar un cierto éxito comunicativo $y$, por tanto, un cierto éxito educacional, sustentado en estudiar cómo los profesores y estudiantes utilizan el lenguaje para construir y compartir conocimiento (Sánchez y Rosales, 2005). 
En la línea de investigación de interacciones comunicativas en salas de clases, Mercer y Howe (2012) evidenciaron que los profesores que utilizan diversas estrategias de comunicación basadas en el uso de tropos retóricos como preguntas abiertas, contribuyen de mejor manera a la participación de los alumnos en la clase, obteniendo resultados de aprendizaje significativamente mejores en el tiempo, a diferencia de lo profesores que no lo hacen. Evidenciaron fundamentalmente que los profesores que utilizan preguntas abiertas para extraer razones y explicaciones de los alumnos respecto al por qué de las respuestas que daban, benefician positivamente la construcción de sus aprendizajes. No sucede así con los profesores que únicamente cautelan revisar las respuestas entregadas por los escolares, inhibiendo un espacio dialógico de impresiones a favor o en contra de la temática tratada (Rosales et al., 2012). Estudios realizados en el ámbito de patrones de discurso en interacciones pedagógicas, han reportado que la mayor frecuencia de preguntas dirigidas a la regulación del flujo de la clase están más orientadas a preguntas de aplicación que de comprensión de contenido. Además, se encontró un fuerte predominio de preguntas con bajo desafío cognitivo, lo que potencialmente conlleva a una menor participación de los estudiantes en los procesos de co-construcción del significado de aprendizaje (Radovic y Preiss, 2010).

Gran parte de la investigación realizada en aulas de clases sitúan la interacción comunicativa como un ámbito fundamental para dar cuenta del aprendizaje alcanzado; sin embargo, la mayoría de estos estudios obedecen a interacciones locales en periodos breves de tiempo. Aun cuando estos hallazgos permiten avanzar en la comprensión sobre cómo los profesores enseñan y cómo los estudiantes aprenden en secuencias comunicativas diádicas y tríadicas, es menester incorporar una medida de la actividad global del sistema de interacción. Un escenario educativo es una red dinámica global que se sustenta históricamente en interacciones locales que van modulando la actividad; sin embargo, el devenir del sistema está sujeto a cambios globales y multivariados que no son posibles de establecer como relaciones univocas restringidas a la acción específica de sus componentes (Scott, 2008).

\subsection{El aula como sistema complejo}

Cuando se hace la pregunta sobre qué es educar y, por tanto, qué es lo connota o describe lo educativo, tendemos a buscar y tomar un conjunto de definiciones teóricas que al final nos conlleva a una polisemia conceptual que difumina el sentido. Desde esta perspectiva, y entendiendo que la educación formal responde a contextos sociales que poseen una teleología implícita basada en objetivos de aprendizaje, aprendizajes esperados y estándares de aprendizaje determinados a priori, se hace visible una definición de lo educativo asociado prescriptivamente a determinismos ambientales que van en contra del devenir del sistema nervioso y por ende la interacción de la vida humana en contextos de comunicación.

Las relaciones que se establecen entre profesoresescolares y escolares-escolares en un espacio dialógico en un aula deben obedecer a configuraciones dinámicas históricas que son propias de un acto educativo que permite distinguirlo de otras acciones, que involucran interacciones humanas, así como también distinciones en sus propios ámbitos. Cuando se pregunta ¿qué distingue un acto educativo de un litigio penal, un baile nacional o un rito particular de una cultura indígena? Todas estas situaciones connotan interacciones humanas específicas, las cuales dados ciertos patrones de comportamiento son posibles relacionarlas con las actividades mencionadas. En el caso del acto educativo, uno podría distinguirlo rápidamente de las situaciones mencionadas anteriormente, a partir de la acción de sus actores y el contexto social en donde se suscita la interacción, así como también el tema en cuestión. Sin embargo, si se planteara la misma pregunta, pero asumiendo ingenuamente que reducimos la complejidad del problema anterior, pensando ahora solo en el propio ámbito de clases educativas, ¿qué describe y connota lo educativo? Lo más probable es que se piense que la distinción sería más fácil y probablemente se encuentren cosas muy similares asociadas a situaciones esperadas producto de la teleología del sistema escolar. Sin embargo, si adicionamos a esa pregunta el conjunto de variables que modulan el sistema de interacción donde se genera el acto educativo, como asignaturas, género de profesores y escolares, metodologías, etnias, niños con capacidades distintas, idioma, contexto socioeconómico, contexto urbano y rural, grado único y multigrado, tipo de evaluación del desempeño docente, etc. ¿se podría distinguir con la misma facilidad un acto educativo de otro? Al parecer no. La reducción conceptual que se ha concebido de lo educativo radica en que no se dimensiona la complejidad del tejido de relaciones que urden en las aulas de diversos contextos. Lamentablemente, el sistema educativo amparado bajo el paragua social y homogeneizante de la escolarización ha desnaturalizado la complejidad del 
acto educativo a un proceso dinámico lineal sustentado en la ideación de metodologías de aprendizaje basadas en el paradigma del procesamiento de la información, sesgando de esta manera la naturaleza dinámica no-lineal de un sistema educativo.

La complejidad de los actos educativos transita desde dinámicas locales propias de los seres humanos que las componen hacia dinámicas globales que integran la interacción de los agentes producto de la coderiva ontogénica. Cada ser humano, como unidad ecológica, está generándose continuamente en la ontogenia producto de la acción de coherencias moleculares, sensoriales, relacionales y viceversa (Maturana y Dávila, 2015). Las interacciones de clases son complejas y exhiben dinámicas de actividad locales a dinámicas de actividad globales moduladas por la mediación semiótica del significado de aprendizaje. La evolución de la comunicación intersubjetiva del aula puede representarse a través de transiciones de fases cuyos estados del sistema dependen de la complejidad de las interacciones, las cuales son de tipo local "bottom up" y de tipo global "top down" (Ibáñez, 2008).

Los sistemas complejos son sistemas en los que su comportamiento colectivo y variabilidad temporal emerge espontáneamente sin ser reducida su dinámica la suma de sus componentes (Valsiner, 2003). El aula es un sistema complejo en la medida que las relaciones que se establecen obedecen a un orden espontáneo correspondiente a múltiples interacciones en unos o varios niveles. El devenir del aula como sistema complejo está asociado a interacciones comunicativas de tipo verbal y gestual, generando primero cambios a nivel local, producto de la acción de un individuo. Luego, a un nivel meso, de interacciones en grupos pequeños de la clase el sistema comienza a exhibir un comportamiento dinámico no-lineal producto de la medición semiótica de conceptos que a su vez implica una co-construcción biológica-cultural (Maturana y Dávila, 2015). Se ha descubierto que conceptos provenientes de la teoría de sistemas de complejos como son: emergencia, autoorganización, incertidumbre, entropía, atractor, punto de acumulación, sinergia, espacios de fase, fractalidad, caos determinista y vaguedad, etc. son atribuibles a sistemas sociales en los cuales la comunicación es un proceso de co-construcción, articulación y transformación del espacio relacional en la cual se urden cerebro, mente, cuerpo y cultura (Ibáñez, 2008).

\subsection{Hacia una formalización de la vida en el aula: nuestra propuesta con fuzzy set}

Para determinar si el aula es un sistema complejo es menester incorporar evidencia empírica que permita atribuir a ciertos comportamientos generados en las interacciones de clases conceptos que son adscritos en la teoría de sistemas complejos (Di Paolo, 2015). Gran parte de la investigación en sistemas complejos proviene de las ciencias aplicadas a dominios de la termodinámica, economía, biología molecular, teoría cuántica, sistema nervioso y climatología. Aun cuando en el área de ciencias sociales se habla de complejidad educativa, no se ha establecido un marco teórico-metodológico lo suficientemente robusto para caracterizar la dinámica de interacciones de clases en el aula como un sistema complejo (Di Paolo y Jaegher, 2012). Disciplinas como la sociología y la econofísica han utilizado técnicas de análisis asociadas a sistema dinámicos no-lineales, como es el caso del exponente de Lyapunov para cuantificar la caoticidad del sistema y tener parámetros de impredictibilidad según la alteración de las condiciones iniciales. En el ámbito de la psicología social, desde la sociogenética se ha avanzado a la comprensión estructural de las comunicaciones integrando elementos de dinámica no-lineal para dar cuenta de la génesis del significado en interacciones comunicativas que involucran marcadores fisionómicos a nivel micro, marcadores del habla a nivel meso, marcadores de coordinaciones en el lenguaje con otros a nivel macro (Valsiner, 2004).

En el ámbito educativo estudios sobre autoorganización del aprendizaje han demostrado que en el aula se generan procesos de interacción que son atribuibles a sistemas complejos. Astudillo et al., (2015) modelaron el comportamiento de autoaprendizaje en estudiantes universitarios a partir de la herramienta de sistemas complejos basada en el análisis topológico de redes sociales. Los resultados evidenciaron, mediante el uso grafos basados en nodos y enlaces asociados a tipos de afinidades y colaboración que, en el aspecto de la afinidad, el grupo evolucionó de una estructura compuesta por tres comunidades a una de dos más densa, entrelazada y cercana. En el aspecto de colaboración para los aprendizajes, se reportó que los mismos individuos conformaron una red de trabajo diluida en enlaces de extensa y homogénea concordancia con las características de una red de colaboración para el aprendizaje eficiente (Astudillo et al., 2015). 


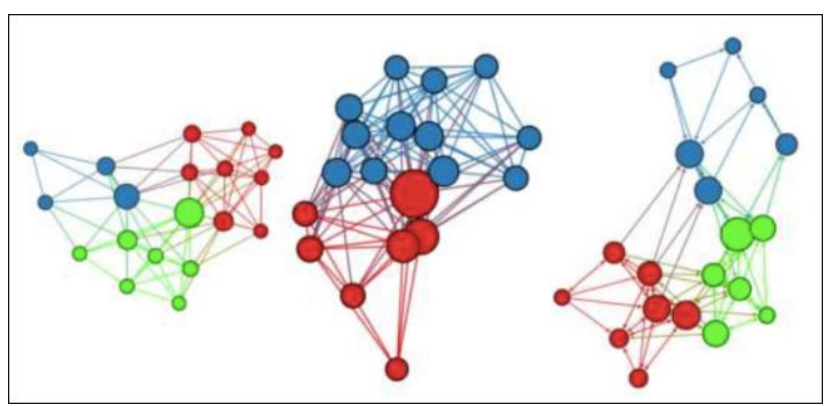

Figura 3: Red topológica de autoaprendizaje por cooperación (imagen extraída de Astudillo et al., 2015).

En la misma línea Mason y Watts (2012) han propuesto el análisis topológico de redes para explicar el aprendizaje, estableciendo una analogía del aula de aprendizajes como un sistema de redes. Los sistemas de redes adquieren complejidad evolutiva en base a ciertos parámetros medibles relevantes; centralidad de intermediación, coeficiente agrupamiento y longitud de camino medio. Aun cuando el análisis de redes permite establecer el comportamiento autoorganizado del sistema, es necesario avanzar hacia una comprensión específica del tipo de enlace o vínculo entre los nodos. Es decir, que los enlaces comprendidos desde la topología de redes están dados por valores numéricos que oscilan, pero que no permiten cuantificar con precisión la incertidumbre de las relaciones en el sistema. Las interacciones que se suscitan en el aula de clases son de complejidad creciente según el nivel de dialogicidad que se despliegue durante la mediación semiótica. La generación espontánea de relaciones que se den en los sistemas complejos suelen ser difusas y vagas, requiriendo mayor especificidad a la hora de cuantificar la incertidumbre y así reducir la entropía del sistema para desentrañar mejor los tipos de relaciones.

Una alternativa de formalización y modelamiento de las interacciones de clases es la teoría de conjuntos difusos o lógica difusa, la cual permite cuantificar la incertidumbre de un sistema donde las relaciones que caracterizan la dinámica son vagas o difusas. Es así como, haciendo uso de teoría fuzzy como medio de representación del conocimiento, Videla, Rossel y Leyton (en prensa) analizaron el carácter emergente de diversos y sutiles patrones de enseñanza, que van progresivamente utilizando los profesores en su afán de comunicar sus saberes a los escolares. Los patrones obtenidos poseían una naturaleza borrosa, difusa, sin fronteras, los cuales emergieron a través del material etnográfico que se utilizó en una primera aproximación cualitativa respecto a las interacciones comunicativas entre una profesora destacada en matemáticas y sus alumnos. Es menester connotar que la ausencia o presencia de estos patrones no es total, sino que más bien obedece a grados de ausencia o presencia.

El concepto de conjunto fuzzy dado por Zadeh, $\mathrm{Fu}$, Tanaka y Shimura (1975), está basado en el modelamiento de la pertenencia parcial en un conjunto para ciertos elementos de un universo dado. En particular, una variable fuzzy lingüística es un atributo cuyo dominio contiene valores lingüísticos, que son rótulos para un subconjunto fuzzy; por ejemplo, el conjunto fuzzy Edad se puede describir como:

Edad $=$ \{bebé, infante, niño, pre_puber, adolescente, adolescente mayor, adulto joven, adulto, adulto mayor, casi anciano, anciano\}.

La Teoría Fuzzy, introducida por Zadeh et al. (1975), permite modelar la incertidumbre y evaluar la entropía de un sistema de relaciones fuzziness de una manera natural, debido a su axiomática, la cual concibe un nuevo tipo de matemática, donde todos los elementos son conceptualizados de manera gradual y no taxativos, como en la matemática clásica; es por esta razón que algunos matemáticos son críticos de esta teoría. Según Córdova y Leyton (2013) el modelamiento se hace tomando en cuenta las siguientes consideraciones:

Sea $\mathrm{X}$ un conjunto clásico de objetos, llamado universo. La pertenencia en un subconjunto A de $\mathrm{X}$ se puede definir en términos de la función característica:

$$
\mu_{\mathrm{A}}: \mathrm{X} \underset{\mathrm{x}}{\stackrel{\mathrm{\mu}}{\mu_{\mathrm{A}}(\mathrm{x})}\{0,1\}}
$$

donde: $\mu_{\mathrm{A}}(\mathrm{x})=\left\{\begin{array}{l}1, \mathrm{x} \in \mathrm{A} \\ 0, \mathrm{x} \notin \mathrm{A}\end{array}\right.$

Si el conjunto de valoración $\{0,1\}$ es extendido al intervalo real $[0,1]$, entonces se puede hablar de pertenencia parcial en $\mathrm{A}$, donde $\mu_{\mathrm{A}}(\mathrm{x})$ es el grado de pertenencia de $\mathrm{X}$ en $\mathrm{A}$ y en que los valores 0 y 1 son interpretados como "no pertenencia" y "pertenencia total" respectivamente. Claramente, A es un subconjunto de $\mathrm{X}$, el cual no tiene fronteras definidas (Kandel, 1982). Esto permite dar la siguiente definición: 
Definición. Sea $X$ un espacio de objetos. Un subconjunto fuzzy A de X está caracterizado por el conjunto de pares:

$\mathrm{A}=\left\{\left(\mathrm{x}, \mu_{\mathrm{A}}(\mathrm{x})\right) / \mathrm{x} \in \mathrm{X}\right\}$, donde $\rightarrow \mu_{\mathrm{A}}: \mathrm{X}[0,1]$

De la definición, se puede ver que en realidad un subconjunto fuzzy A de $\mathrm{X}$ es una función de $\mathrm{X}$ en $[0,1]$ y es una generalización del concepto clásico de subconjunto. Un concepto fuzzy puede ser representado por un conjunto fuzzy y sus conceptos relativos:

i) Dos conjuntos fuzzy $\mathrm{A}$ y $\mathrm{B}$ se dicen iguales, denotado por $A=B$, ssi

$$
\forall \mathrm{x} \in \mathrm{X}, \mu_{\mathrm{A}}(\mathrm{x})=\mu_{\mathrm{B}}(\mathrm{x}) .
$$

ii) Dados A y B conjuntos, A es subconjunto de B, denotado por $A \underline{C} B$, ssi

$$
\forall \mathrm{x} \in \mathrm{X}, \mu_{\mathrm{A}}(\mathrm{x})<\mu_{\mathrm{B}}(\mathrm{x}) .
$$

iii) Un conjunto fuzzy A se dice normal si existe $\mathrm{x} \in$ $\mathrm{X}$ tal que

$$
\mu_{\mathrm{A}}(\mathrm{x})=1
$$

iv) El complemento A de un conjunto fuzzy $\mathrm{A}^{\mathrm{c}}$ está caracterizado por:

$$
\mu_{\mathrm{A}}(\mathrm{x})=1-\mu_{\mathrm{A}}(\mathrm{x}), \forall \mathrm{x} \in \mathrm{X} .
$$

v) La unión $A U B$ de dos conjuntos fuzzy A y B, está caracterizada por:

$$
\mu_{\mathrm{AUB}}(\mathrm{x})=\max \left(\mu_{\mathrm{A}}(\mathrm{x}), \mu_{\mathrm{B}}(\mathrm{x})\right), \forall \mathrm{x} \in \mathrm{X}
$$

vi) La intersección $A \cap B$ de dos conjuntos fuzzy A y $B$, está caracterizada por

$$
\mu_{\mathrm{A} \cap \mathrm{B}}(\mathrm{x})=\min \left(\mu_{\mathrm{A}}(\mathrm{x}), \mu_{\mathrm{B}}(\mathrm{x})\right), \mathrm{V} \mathrm{x} \in \mathrm{X} .
$$

Observación: Es posible constatar que $\mathrm{A} \mathrm{U} \mathrm{A}^{\mathrm{c}} \neq \mathrm{X}, \mathrm{y}$ que $\mathrm{A} \cap \mathrm{B} \neq \varnothing$

En general estos postulados son muy importantes y no suceden en la clásica lógica bivaluada. Esto se debe a la naturaleza de los conjuntos fuzzy, en que sus fronteras no están claramente definidas.

vii) Si $A_{1}, A_{2}, \ldots, A_{n}$ son subconjuntos fuzzy de $X_{1}, X$ 2,..., $X_{n}$ respectivamente, el producto cartesiano $A_{1} X$ $\mathrm{A}_{2} \mathrm{X} \ldots . . . \mathrm{X} \mathrm{A}_{\mathrm{n}}$ es definido como un subconjunto fuzzy de $\mathrm{X}_{1} \mathrm{x} \mathrm{X}_{2} \mathrm{x} \ldots . . \mathrm{X}_{\mathrm{n}}$, y está caracterizado por:

$\mu_{\mathrm{A} 1} \quad \mathrm{x} \quad \mathrm{A} 2 \quad \mathrm{x} \ldots \mathrm{x} \quad \operatorname{An}\left(\mathrm{x}_{1}, \mathrm{X}_{2}, \ldots, \mathrm{X}_{\mathrm{n}}\right)=\min \left(\mu_{\mathrm{A} 1}\left(\mathrm{x}_{1}\right)\right.$, $\left.\mu_{\mathrm{A} 1}\left(\mathrm{x}_{2}\right), \ldots, \mu_{\mathrm{An}}\left(\mathrm{x}_{\mathrm{n}}\right)\right\}, \forall\left(\mathrm{x}_{1}, \mathrm{x}_{2}, . ., \mathrm{x}_{\mathrm{n}}\right) \in \mathrm{X}_{1} \mathrm{x} \mathrm{X}_{2}, \mathrm{X} . . \mathrm{x} \mathrm{X}_{\mathrm{n}}$.

viii) Sean $\mathrm{X}_{1}, \ldots, \mathrm{X}_{\mathrm{n}} \mathrm{n}$ universos. Una relación_R en $\mathrm{X}_{1} \mathrm{X} \ldots \mathrm{x} \mathrm{X}_{\mathrm{n}}$ es un subconjunto fuzzy de $\mathrm{X}_{1} \mathrm{X} \ldots \mathrm{X} \mathrm{X}_{\mathrm{n}}$.

Las relaciones fuzzy permiten modelar términos fuzzy tales como: "cercanos", "débilmente", "medianamente", "fuertemente".

ix) Una regla de producción fuzzy es aquella que tiene la forma IF X es A THEN Y es B, X e Y variables fuzzy, A y $\mathrm{B}$ rótulos fuzzy en universos $\mathrm{U}$ y $\mathrm{V}$ respectivamente.

Una regla de producción fuzzy puede ser representada por una relación fuzzy:

$$
\mathrm{R}_{\mathrm{m}}(\mathrm{A}, \mathrm{B})=(\mathrm{AxB}) \mathrm{U}(\mathrm{AxV}) .
$$

$x$ E) Entropía: la entropía sirve para evaluar cuan difuso es un conjunto difuso, es decir poseer medidas de difucidad. Si $X$ es finito la entropía se calcula de la siguiente manera:

$$
e(A)=\sum_{x \in X}(\propto A(x) \cdot \log (\propto A(x)))+(1 \quad \propto A(x) \cdot \log (1 \quad \propto A(x)))
$$

A partir de lo anterior, la función logaritmo solo está definida para los reales mayores que cero. Notar que la sumatoria se hace solo sobre los $x$ que pertenecen a $X$. Por lo tanto, los $x$ que la función $\mu A(x)$ asigna 0 no son tomados en cuenta, ya que no pertenecen.

La utilización de este modelamiento en el ámbito educativo de interacciones de aprendizaje, permitió obtener patrones no necesariamente determinísticos ni lineales, sino más bien encontrar "grados" de causa y efecto entre uno o varios conceptos de $\mathrm{X}$ con otros conceptos Y (Gupta, Ragade, Yager, 1979). Por ejemplo, para dar cuenta de una relación difusa, se obtuvieron los códigos; recapitulación temática, pregunta clave que intenciona el objetivo de la clase e inferencias lógica, los cuales emergieron a partir de la codificación abierta de los corpus etnográficos de observaciones de interacciones de aprendizaje. Un ejemplo que puede ser clarificador de una interacción local modelada por fuzzy set se puede expresar de esta manera:

Recapitulación temática es causa de pregunta clave que intenciona el objetivo de la clase que contiene a inferencia lógica y además está \{Fuertemente o Medianamente\} relacionado con activación de conocimiento previo.

\section{O, expresado de otra manera: $31 \Rightarrow 25 \supset 19^{\wedge} 25 \Re 1$}

$\mathrm{Si}$ deseamos obtener la activación de conocimiento previo, conteniendo además a inferencia lógica con pregunta clave que intenciona el logro del objetivo de la clase, es adecuado emplear \{Fuertemente o Medianamente\} recapitulación temática".

Es claro que se puede inferir que mientras mejor se realice la recapitulación temática pasa a ser una causa más potente para obtener activación de conocimiento. Este tipo de herramienta de modelamiento y formalización de relaciones difusas permite cuantificar la incertidumbre de un sistema de interacción de clases entre profesores y escolares a través del establecimiento de grados de relaciones que pueden oscilar infinitamente entre una 0 y 1 y no se restringe a 0 o 1 . Además al cuantificar la 
incertidumbre del sistema se disminuye la entropía y la comprensión de relaciones dentro del sistema se hace más plausible debido a que disminuye el volumen y la difucidad de las relaciones que se gestan. Por lo demás, es claro que las relaciones sociales construidas al interior de sistema de interacción de clases son complejas en el sentido que involucran un conjunto de variables asociadas a la mediación semiótica de los conceptos a desarrollar. Avanzar hacia la formalización de la organización de la vida en el aula permite no solo incorporar evidencia empírica a la idea del aula como sistema complejo, sino también, contribuye a connotar y definir qué es lo educativo y de qué manera la dinámica que exhiben los sistemas de interacción de aprendizaje en el aula podría reconfigurar el concepto de educación hacia un enfoque relativista y contextual propios de la teoría de sistemas enmarcadas en el paradigma de la complejidad.

\section{REFERENCIAS}

Astudillo, H., Navarrete, M ., Jara, C., y Faúndez, C. (2015). Evidencia de Auto Aprendizaje como Manifestación de un Comportamiento Colectivo Espontáneo en el Aula. Formación Universitaria, 8(1).

Córdova, F., y Leyton, G. (2013). A Fuzzy Control Heuristic Applied Non Linear Dynamic Systems Using a Fuzzy Knowledge Representation. International Journal of Computers Communications \& Control, 8(1), pp. 50-60.

Di Paolo, E. (2015). Interactive time-travel: On the intersubjective retromodulation of intentions, Journal of Consciousness Studies, 22, 49-74.

Di Paolo, E. y De Jaegher, H. (2012). The interactive brain hypothesis. Frontiers in Human Neuroscience, 6, 163.

Fossa, Cornejo, C., y Carré, D. (2016). Análisis semiótico en secuencias de interacción empáticas y no empáticas: Un estudio microgenético. Revista de Psicología USP, 27(3), 441- 449.

Froese, T. (2010). From cybernetics to second-order cybernetics: A comparative analysis of their central ideas. Constructivist Foundations, 5(2), 75-85.

Froese, T. (2011). Breathing new life into cognitive science. Avant. The Journal of the PhilosophicalInterdisciplinary Vanguard, 2(1), 95-111.

Froese, T., y Fuchs, T. (2012). The extended body: A case study in the neurophenomenology of social interaction. Phenomenology and the Cognitive Sciences, 11(2), 205235.

Gupta, M., Ragade, R., y Yager, R. (1979). Advances in Fuzzy Set Theory and Applications. North-Holland.

Ibáñez, A. (2008). Dinámica de la cognición. Editorial: Comunicaciones Noreste Limitada.

Kandel, A. (1982). Fuzzy Techniques in Pattern Recognition, Wiley-Interscience.

Mason, W., y Watts, D. (2012). Collaborative learning in networks, Proceedings of the National Academy of Sciences, 109(3), 764-769.

Maturana, H., y Varela, F. (1973). De máquinas y seres vivos: una teoría sobre la organización biológica. Santiago: Editorial Universitaria.
Maturana, H., Mpodozis, J., y Letelier, J. C. (1995). Brain, language and the origin of human mental functions. Biol Res., 28(1), 15-26.

Maturana, H. R., y Mpodozis, J. (2000). The origin of species by means of natural drift. Revista Chilena de Historia Natural, 73, 261-310.

Maturana, H. R. (2011). Ultrastability ... autopoiesis? Reflective response to Tom Froese and John Stewart. Cybernetics \& Human Knowing, 18(1-2), 143-152.

Maturana, H., y Dávila, X. (2015). El árbol del vivir. Santiago de Chile: MvP Editores.

Mercer, N. (1997). La construcción guiada de conocimiento: el habla de profesores y alumnos. Barcelona: Paidós.

Mercer, N. (2001). Palabras y mentes: cómo usamos el lenguaje para pensar juntos. Barcelona: Paidós.

Mercer, N. (2007). Dialogue and the development of children's thinking: a sociocultural approach. London: Routledge.

Mercer, N. (2010). The analysis of classroom talk: Methods and methodologies. British Journal of Educational Psychology, 80, 1-14.

Mercer, N., y Howe, C. (2012). Explaining the dialogic processes of teaching and learning: The value and potential of sociocultural theory. Learning, Culture and Social Interaction, 1, 12-21.

Radovic, D., y Preiss, D. (2010). Patrones de discurso observados en el aula de matemática de segundo ciclo básico en Chile. Psykhe, 19(2), 65-79.

Rosales, J., Vicente, S., Chamoso, J., Núñez, D., y Orrantia, J. (2012). Teacher-student interaction in joint word problem solving. The role of situational and mathematical knowledge in ordinary classrooms. Teaching and Teacher Education, 28, 1185-1195.

Sánchez, E., y Rosales, J. (2005). La práctica educativa. Una revisión a partir del estudio de la interacción profesoralumnos en el aula. Cultura y Educación, 70(2), 147173.

Scott, P. (2008). Talking a way to understanding in science classrooms. In N. Mercer, y S. Hodgkinson (Eds.), Exploring talk in school (pp. 17-36). London: Sage.

Thompson, E., y Cosmelli, D. (2011). Brain in a Vat or Body in a World? Brainbound versus Enactive Views of Experience. Philosphical Topics,39(1).

Valsiner, J. (2002). Forms of dialogical relations and semiotic autoregulation within the self. Theory \& Psychology, 12(2), 251-265.

Valsiner, J. (2003). Beyond social representations: a theory of enablement. Papers on Social Representations, 12, 7.17.16

Valsiner, J. (2004). Semiotic autoregulation: dynamic sign hierarchies constraining the stream of consciousness. Paris, França: Sage.

Varela, F., Maturana, H., y Uribe, R. (1974). Autopoiesis: The organization of living systems, its characterization and a model. Biosystems, 5, 187-196.

Varela, F. (1980). Describing the logic of the living In: M.Zeleny (Ed.), Autopoeisis: A theory of the living organization (pp.36-48). New York: North-Holland.

Videla, R., Leyton, G., y Rossel, S. (en prensa). Análisis de la interacción de clases de profesores destacados en matemáticas: aproximación desde un estudio de caso en Chile. Revista Cultura y Educación.

Zadeh, L., Fu, K., Tanaka, K., y Shimura, M., (1975). Fuzzy Sets and their Applications to Cognitive and Decisions Processes. Academic Press, Inc. 